\title{
Leakage effects of dominant and higher order modes on slotline
}

\section{Jan Macháč, Jan Zehentner}

\author{
Faculty of Electrical Engineering, \\ Czech Technical University Technická 2, \\ 16627 Prague 6, Czech Republic.
}

\begin{abstract}
The properties of the slotline when the dominant bound or leaky wave propagates, together with the behaviour of new higher order modes are investigated. The frequency range in which the slotline transmits the bound wave only is determined and the closed-form formula for its upper cutoff frequency is presented. The evolution of the propagation constant in the spectral gap between the propagating bound and leaky wave regions is shown. The influence of the substrate losses on leaky wave behaviour is presented. Some new aspects of both space and surface higher order leaky waves on the wide-slot line are discussed.
\end{abstract}

\section{Introduction}

Uniplanar structures are very widely used in millimetrewave circuits and monolithic MICs. This concerns above all the coplanar waveguide, coplanar strips and slotline. A number of research groups have investigated the behaviour of these transmission lines and modifications to them (sidebounded substrate, conductor-backed lines) within a wide frequency band. Many papers dealing with this topic have been published recently. The slotline has been investigated mostly within the frequency range where only the bound wave is transmitted $[1,2,3]$. Bound higher order waves have been described in $[4,5]$ while surface and space leaky waves are described generally in [6,7]. Shigesawa et al [8] revealed simultaneous propagation of both bound and leaky waves on open lines. A spectral gap between the bound and leaky wave regime was discussed in [9]. The slotline itself is analysed in $[10,11]$. Oliner sums up the latest findings regarding the performance of printed circuits in [6].

Leaky waves degrade the characteristics of circuits. They cause energy leakage from the line, crosstalk between circuits, dispersion of short transmitted pulses and resonant effects when circuits are closed in a package. A layered substrate has been proposed [12] for suppressing substrate leakage . Alternatively a side-nonhomogeneous substrate can be used [13].

This paper contributes to discussion about new effects occurring on the slotline, and comprises a detailed investigation of the slotline in the microwave and millimetre-wave bands, surface leaky waves, the evolution of a spectral gap, the existence of space leaky waves, and an analysis of higher bound and leaky waves.

The slotline explored by the spectral domain method is assumed to be homogeneous on the boundless dielectric substrate, which may be lossy. Fig. 1 shows its cross-section. Zero thickness metallization with ideal conductivity is also assumed.

The frequency range in which only the dominant bound wave propagates is determined. A new closed-form formula enables us to calculate its upper limit. The evolution of a spectral gap caused by slot width increase is shown. First, the influence of substrate losses on leaky waves is examined. In addition to [10] a new insight into the behaviour of higher order modes on the wide-slot line is given, both at low frequencies when space leaky waves are excited and in the higher frequency region where surface leaky waves occur.

\section{Dominant Bound Wave on a Narrow Slotline}

For a lossless line at low frequencies, propagation constant $\beta r$ of the fundamental mode is real and is higher than $k_{T M O}$ which belongs to the dominant surface mode $\mathrm{TM}_{0}$ (Fig. 2) so that the leakage condition (6) is not fulfilled. Consequently the wave is bound and propagates along a line with constant amplitude. At the frequency denoted $f_{c}$ in Fig. 2 these propagation constants are equal. This is the upper cut-off frequency up to which only the dominant bound wave propagates. Above this frequency the bound wave converts into a leaky wave, leaking energy into the substrate. Values $f_{c}$ versus $\left(h * \sqrt{\varepsilon_{r}}\right)$ are plotted in Fig. 3 where $h$ and $\varepsilon_{\mathrm{r}}$ are substrate thickness and permittivity, respectively.

A set of computed data $f_{c}$ was fitted by the least squares method to the closed-form formula

$$
\mathrm{f}_{\mathrm{c}}=\frac{\mathrm{A}_{1}}{\left(\mathrm{~h} \sqrt{\varepsilon_{\mathrm{r}}}\right)^{\mathrm{A}_{2}}}+\mathrm{A}_{3} \quad[\mathrm{GHz}, \mathrm{mm}],
$$

where coefficients $A_{1}, A_{2}, A_{3}$ are functions of the slotline width $\mathrm{w}$ set in $\mathrm{mm}$

$$
\begin{aligned}
& A_{1}=134.6-0.3 w^{3.8}-\frac{5}{w^{0.5}}+\quad \text { (2) } \\
& \frac{0.09}{0.009+|w-0.37|^{3}}+\frac{0.35}{(0.8|w-0.7|+0.9|w-1|)^{1.7}}
\end{aligned}
$$

$$
\mathrm{A}_{2}=0.88+\frac{0.6}{(w+1.35)^{2.8}}+\frac{0.004}{(|w-0.4|+|w-1|)^{2}}
$$

$$
\begin{aligned}
A_{3}=-1.07 & -0.9 w^{1.09} \\
& +\frac{0.928}{(w+0.77)^{10.5}}+\frac{0.009}{0.14+|w-1.51|^{9}}
\end{aligned}
$$

The validity of these formulae is within the interval

$$
0.693+1.23 w^{1.95}-0.182 w^{4.079} \leq h \sqrt{\varepsilon_{r}} \leq 7.6
$$

The weak sensitivity of the cut-off frequency to slot width changes is clearly shown in Fig. 3. 


\section{Dominant Surface Leaky Wave}

Just above $\mathrm{f}_{\mathrm{c}}$ a spectral gap occurs, as described recently by Oliner and Jackson [9]. Its evolution for a high permittivity substrate with increasing slot width is shown in Fig. 4. The propagation constant above $f_{c}$ remains real, but the corresponding wave is non-physical since its amplitude is constant along the line while it increases in direction $\mathrm{x}$. This branch of the curve is even doublevalued. At frequency $f_{1}$ and above (Fig. 2) the propagation constant becomes complex. Between $f_{1}$ and $f_{2}$ the corresponding wave is non-physical and cannot be excited since condition (6) is not fulfilled. Above frequency $f_{2}$ the physically real leaky wave is excited because the leakage condition

$$
\beta \mathrm{r}<\mathrm{k}_{\mathrm{TMO}}
$$

is valid. For a wider slot the branch of a real propagation constant is singlevalued while the real part of the complex propagation constant (e.g. for $w=2.088 \mathrm{~mm}$ ) becomes doublevalued with the upper non-physical branch, Fig. 4.

The amplitude of the leaky wave decreases along the line due to the energy leakage and increases in direction $x$. A simple two plane wave model [10] describes this wave. According to this model the leaky wave can be composed of two plane waves, one propagating to the left and one to the right, aside from the plane of symmetry. Substrates exhibit losses. These losses, accounted for by the imaginary part $\varepsilon_{r i}$ of the complex dielectric constant, cause an additional decrease in the field along the line. The calculated attenuation constant in both bound and leaky wave regions in dependence on $\varepsilon_{\mathrm{ri}}$ is shown in Fig. 5. The influence of the substrate losses on the phase constant is negligible, and is therefore not shown. The influence of substrate losses in comparison with losses caused by energy leakage into the substrate is of minor importance only, Fig. 5. Due to the substrate losses the field amplitude grows less steeply in the $x$ direction (Fig. 1) than in the lossless case [10]. This effect can be disregarded for low-loss substrates.

\section{Bound and Leaky Higher Order Modes on a Wide Slotline}

Higher order modes propagating along the slotline with a wide slot have been described in [10]. Even and odd modes, both bound and leaky, were detected. The crucial point in the search for surface and space leaky waves is the choice of the proper integration path in the complex plane, as the integrand may have a limited number of poles and two branch points [7]. Unlike [10], we have now used the integration path deformed around the branch points, Fig. 6. This path lies partly on the improper sheet of Riemann's plane, dashed in Fig. 6, and is responsible for growth of the field amplitude in direction $y$, perpendicular to the substrate. Even and odd space leaky waves with amplitudes increasing simultaneously in directions $\mathrm{x}$ and $\mathrm{y}$ have been obtained. The propagation constant of the even modes is shown in Fig. 7a. $E_{0}$ and $E_{1}$ denote fundamental and first even bound modes. The higher order surface leaky waves behave similarly as the fundamental mode on the narrow-slot line, Fig. 7b. The attenuation constants of these waves are not shown in Fig. $7 \mathrm{~b}$ owing to their very low magnitudes. The spectral gaps of these waves are narrow. The propagation constant of the space leaky wave shown in Fig. 7a is not reproduced in Fig. 7b. Characteristics of the odd higher order modes are shown in Fig. 8 where $\mathrm{O}_{1}, \mathrm{O}_{2}$ denote the first and second bound modes. Their behaviour is similar to that of the even modes.

Space leaky waves excited by the wide slotline (Fig. 7a, 8a) leak energy not only to the space above and below the substrate but also into the substrate itself. Even modes radiate into four lobes, two above and two below the substrate, each declined identically from the slot axis. Odd modes are more favoured for potential applications than even modes since they have only one lobe on each side of the substrate [10].

\section{Conclusions}

The transmission characteristics of the uniform slotline were studied both in bound and leaky wave regions. The upper cut-off frequency of the fundamental bound wave was determined, and a closed-form formula was achieved which facilitates its calculation. Just above it, a spectral gap occurs within which the line transmits no wave. The gentle structure and variation of the propagation constant inside this gap in dependence on the slot width for a high permittivity substrate has been shown. The influence of substrate losses on leaky wave behaviour has been examined. For customary low-loss substrates this influence is insignificant. Higher order space and surface even and odd leaky waves on the wide-slot line were analysed in detail. Space leaky waves were detected at low frequencies. The behaviour of surface leaky waves at high frequencies is similar as in the case of the fundamental mode on a narrow slotline. This knowledge can be applied in the design of a wide-slot leaky wave antenna.

\section{Acknowledgement}

This work was partly done on the SP-2 computer at the Joint Supercomputer Center of the Czech Technical University, University of Chemical Technology and IBM (Prague). The authors thank Prof. W. Menzel, University of Ulm, for the analysis done partly on his Department's computers.

\section{References}

[1] Itoh T., Mitka R.: Dispersion characteristics of slot lines, Electronics Letters, vol. 7, No. 13, 1971, pp.364-365.

[2] Knorr J.B., Kuchler K.D.: Analysis of coupled slots and coplanar strips on dielectric substrate, IEEE Trans. on Microwave Theory and Techn., vol. MTT-23, No. 7, 1975, pp. 541-548.

[3] Rozzi T., Moglie F., Morini A., Marchionna E., Politi M.: Hybrid modes, substrate leakage and losses of slot line at millimeter-wave frequencies, IEEE Trans. on Microwave Theory and Techn., vol. MTT-38, No. 8,1990,pp.1069-1078.

[4] Citerne J., Toutain S., Raczy L.: Fundamental and higher order modes in microstrip lines, Proc. of the 5th EuMC, Hamburg, Sept. 1975, pp. 273-277

[5] Mejsnar K.: Higher order modes on slot lines, Proc. of the 10th EuMC, Warsaw, Sept. 1980, pp. 327-331

[6] Oliner A.A.: New leakage effects and new dominant modes in millimeter-wave printed circuits, Proc of the $23 \mathrm{rd}$ EuMC, Madrid, Sept. 1993, pp. 49-51

[7] Das N.K., Pozar D.M.: Full-wave spectral-domain computation of material, radiation, and guided wave losses in finite multilayered printed transmission lines, IEEETrans. on Microwave Theory and Techn., vol. MTT-39, No. 1, 1991, pp. 54-63

[8] Shigesawa H., Tsuji M., Oliner A.A.: Simultaneuos 
propagation of bound and leaky dominant modes on printed circuit lines: a ñew general effect, IEEE MTT-S Digest, 1995, pp. 145-148.

[9] Oliner A.A., Jackson D.R.: On spectral gaps at the transition between bound and leaky modes, Proc. of the 15 th International Symposium on Electromagnetic Theory, URSI, St.Petersburg, Russia, May 1995, pp. 764-766.

[10] Machác J., Zehentner J.: Leaky waves on a slot line, Proc. of the 15th International Symposium on Electromagnetic Theory, URSI, St.Petersburg, Russia, May 1995, pp. 761-763.
[11] Machác J., Zehentner J.: Utilizable frequency band and leaky wave phenomenon of the slotline, Proc. of the 11th International Microwave Conference MIKON 96, Warsaw, Poland, May 1996, pp. 193-i97

[12] Lin Y., Itoh T.: Control of leakage in multilayered conductor-backed coplanar structures, 1994 IEEE MTT-S Digest, pp. $141-144$

[13] Das N.K.: Two conductor-backed configuration of slotline or coplanar waveguide for elimination or suppression ofthe power-leakage problem, 1994 IEEE MTTS Digest, pp. 154-156

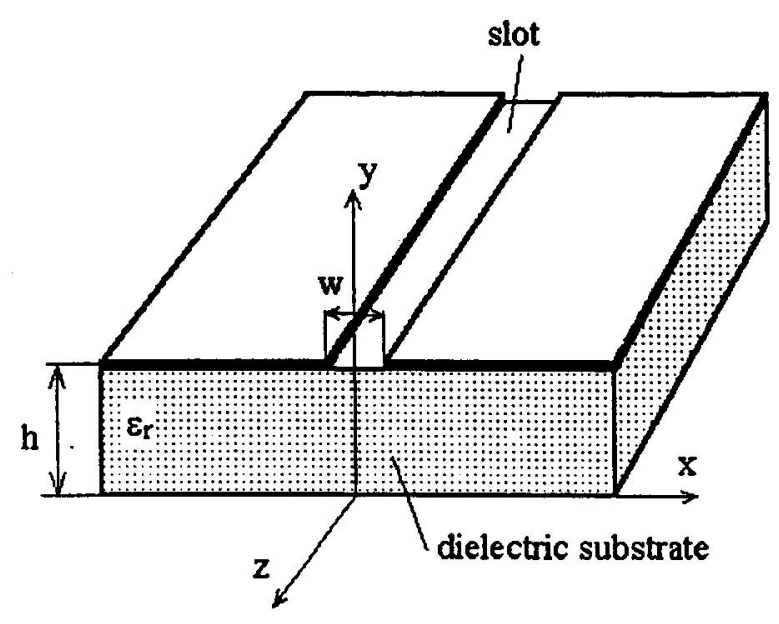

Fig. 1 Cross-section of the slotline

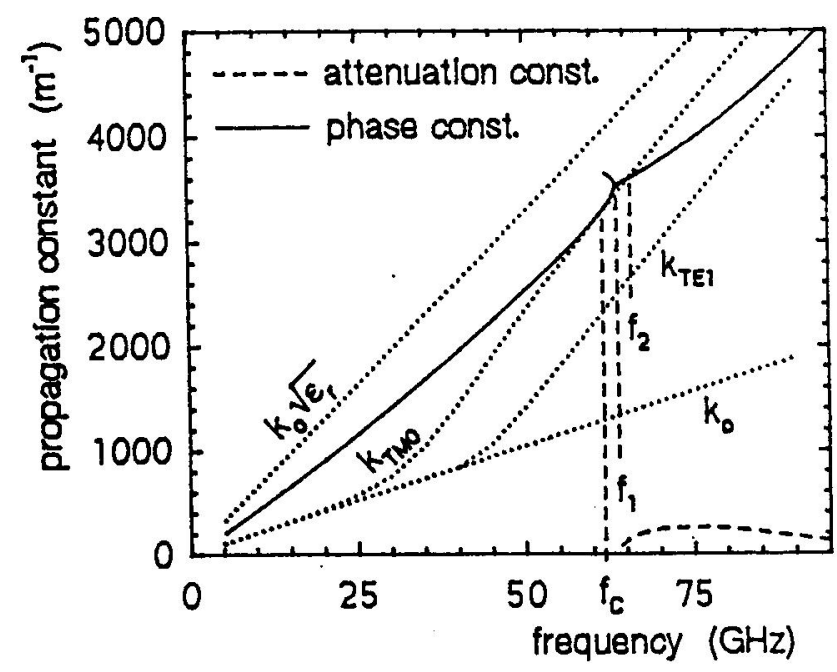

Fig. 2 Propagation constant of the slotline $\varepsilon_{\mathrm{r}}=9.8, \mathrm{~h}=0.635 \mathrm{~mm}, \mathrm{w}=0.1 \mathrm{~mm}$ 


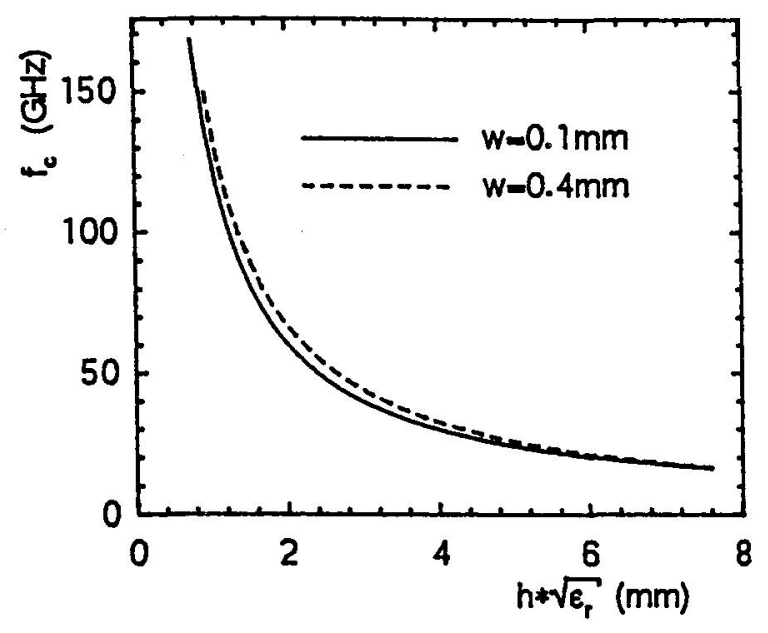

Fig. 3 Upper cut-off frequency of the slotline

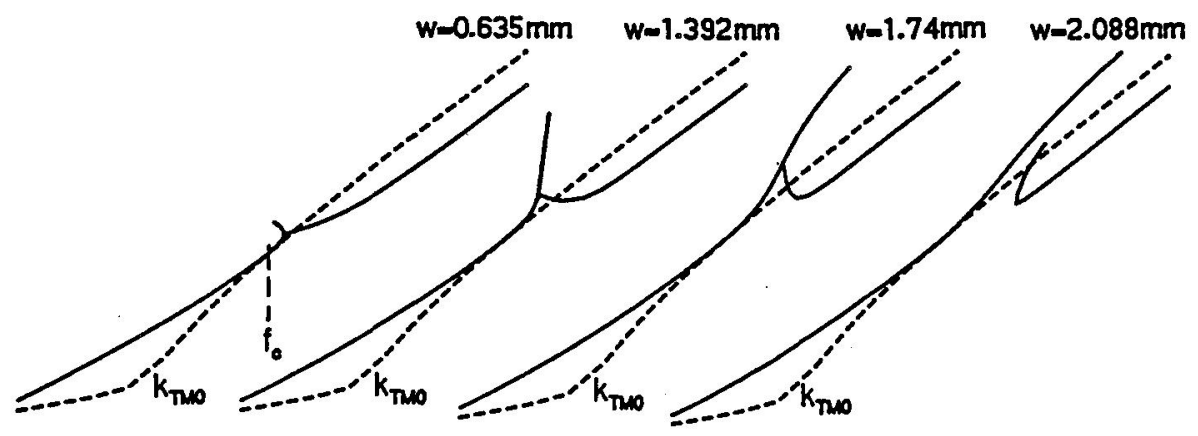

Fig. 4 Evolution of the spectral gap in dependence on the slot width $w, \varepsilon_{\mathrm{r}}=20, \mathrm{~h}=3.48 \mathrm{~mm}$

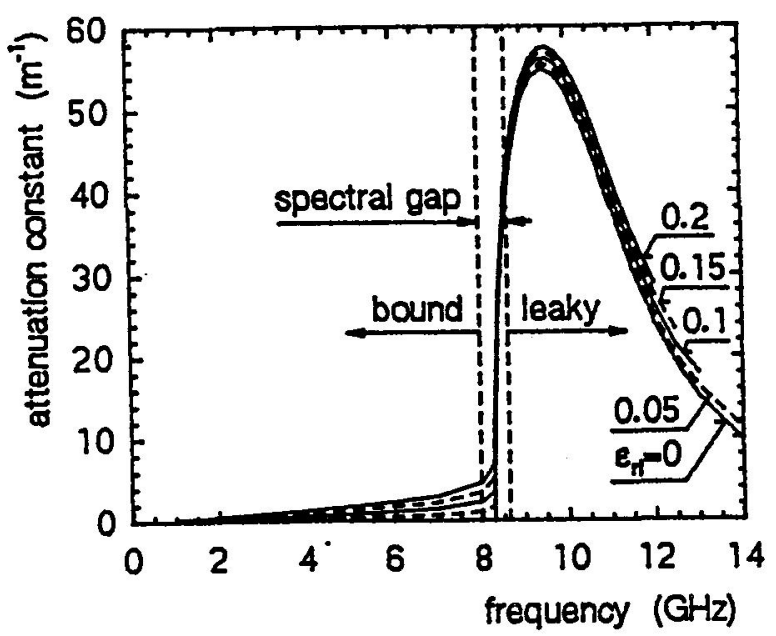

Fig. 5 Attenuation constant of the slotline given in Fig. 4 in dependence on the substrate loss factor, $w=0.635 \mathrm{~mm}$

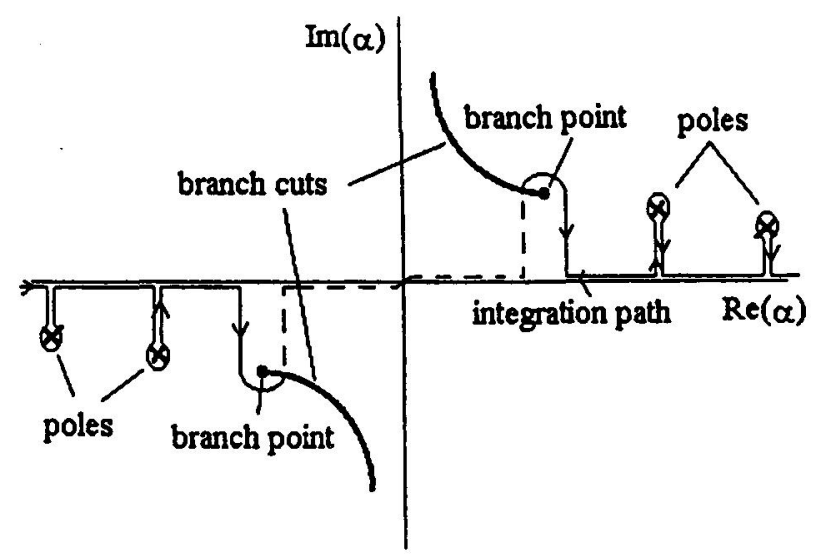

Fig. 6 Integration path in the complex plane 


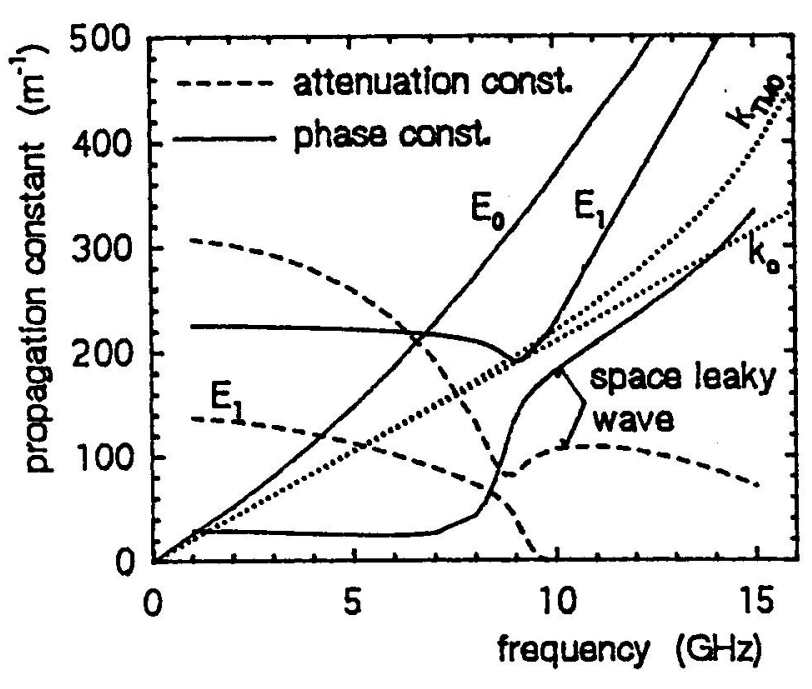

a

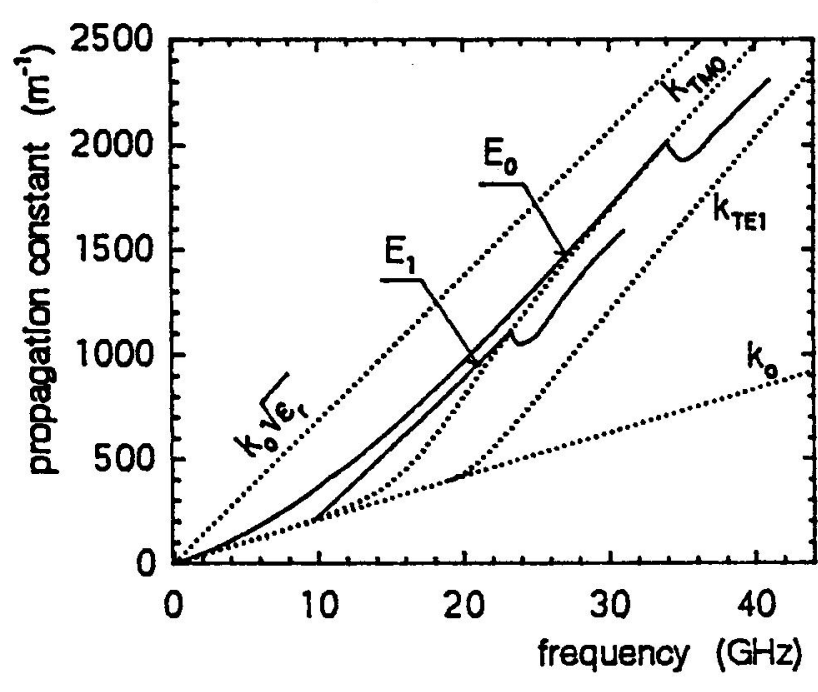

b

Fig. 7 Propagation constant of even higher order modes on the slotline $\varepsilon_{\mathrm{r}}=11, \mathrm{~h}=1.27 \mathrm{~mm}$, $\mathrm{w}=20 \mathrm{~mm}$; a) at low frequencies - space leaky waves, b) at high frequencies - surface leaky waves

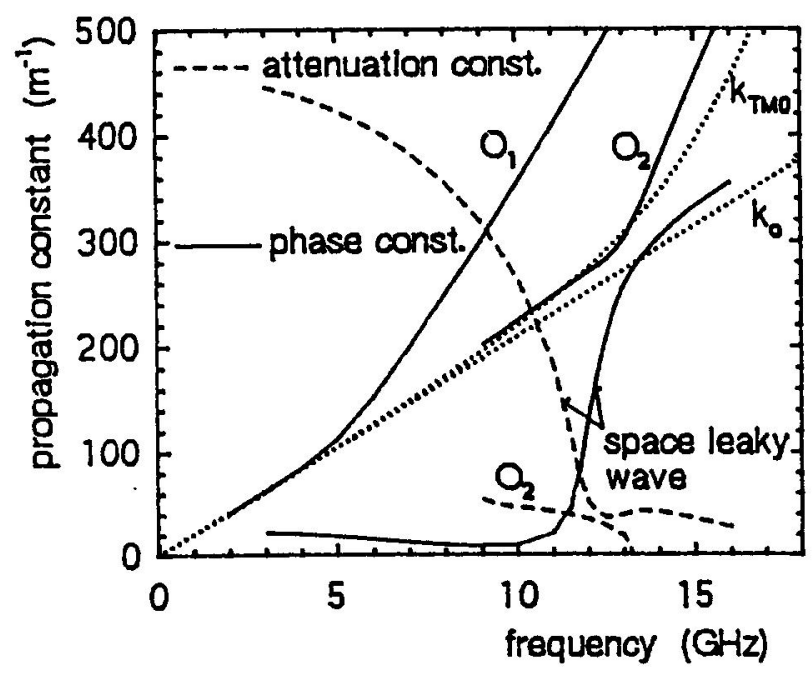

a

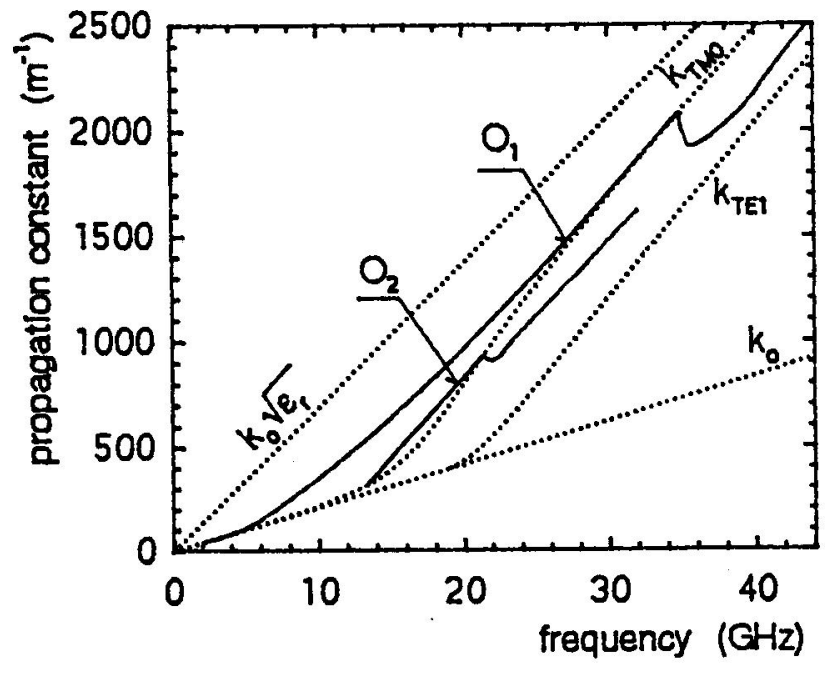

b

Fig. 8 Propagation constant of odd higher order modes on the slotline specified for Fig. 7;

a) space leaky wave, b) surface leaky waves 